\title{
A Comparison of Aerobic Fitness Testing on a Swim Bench and Treadmill in a Recreational Surfing Cohort: A Pilot Study
}

\author{
Hamzeh Khundaqji ${ }^{1}$ (D), Enad Samain ${ }^{1}$, Mike Climstein ${ }^{1,2,3}{ }^{\mathbb{D}}$, Ben Schram ${ }^{1}{ }^{\mathbb{D}}$, Wayne Hing ${ }^{1(\mathbb{D})}$ \\ and James Furness 1 ,* \\ 1 Water Based Research Unit-Bond Institute of Health and Sport, Bond University, Gold Coast, QLD 4226, \\ Australia; hamzeh.khundaqji@student.bond.edu.au (H.K.); enad.samain@student.bond.edu.au (E.S.); \\ michael.climstein@scu.edu.au (M.C.); bschram@bond.edu.au (B.S.); whing@bond.edu.au (W.H.) \\ 2 School of Health and Human Sciences, Southern Cross University, Lismore, NSW 2480, Australia \\ 3 Exercise, Health and Performance Faculty Research Group-Faculty of Health Sciences, \\ The University of Sydney, Sydney, NSW 2006, Australia \\ * Correspondence: jfurness@bond.edu.au; Tel.: +617-5595-3354
}

Received: 4 April 2018; Accepted: 9 June 2018; Published: 11 June 2018

\begin{abstract}
The intermittent manner of surfing accentuates the importance of both the aerobic and anaerobic energy systems. Currently, the optimal method of assessing surfing-specific aerobic fitness is using a swim bench (SWB) ergometer; however, their limited availability presents a barrier to surfers wanting to know their maximal aerobic power $\left(\mathrm{VO}_{2 \text { peak }}\right)$. As a result, the aims of this pilot study were to determine the $\mathrm{VO}_{2 \text { peak }}$ of recreational surfers using a new commercial SWB ergometer and to propose and examine the feasibility of a regression model to predict $S W B$ ergometer $V_{2}$ peak values. A total of nine recreational surfers were assessed where body measurements were conducted followed by maximal aerobic capacity testing (swim bench and treadmill) to profile the cohort. Findings demonstrated that $\mathrm{VO}_{2 \text { peak }}$ values were significantly greater $(p<0.001)$ on the treadmill compared to the SWB ergometer $(M=66.01 \pm 8.23$ vs. $37.41 \pm 8.73 \mathrm{~mL} / \mathrm{kg} / \mathrm{min})$. Peak heart rate was also significantly greater on the treadmill compared to the SWB ergometer. Multiple regression analysis was used to produce a model which predicted $S W B V_{2 \text { peak }}$ values with an $R^{2}$ value of 0.863 and an adjusted $\mathrm{R}^{2}$ value of 0.726 . The physiological profiling of the recreational cohort coupled with a surfer's predicted $\mathrm{SWB} \mathrm{VO}_{2 \text { peak }}$ value will allow for identification of surfing-specific aerobic fitness levels and evidence-based training recommendations.
\end{abstract}

Keywords: aerobic fitness; aerobic power; $\mathrm{VO}_{2 \text { peak }} ; \mathrm{VO}_{2 m a x}$; ergometer; treadmill

\section{Introduction}

Surfing is regarded as part of a lifestyle and culture for those living on the coastal borders of countries like Australia and the United States. Today, surfing is enjoyed as an iconic pastime by 2.7 million Australians and 37 million individuals worldwide [1,2]. With its growth into a global multi-million-dollar industry and its recent induction into the Olympics, it is reasonable to assume that surfing's impressive growth over the last decade will continue [3].

Using time-motion analysis, surfing has been subdivided into intermittent periods of arm paddling, prolonged periods of rest, and wave riding [3]. Monitoring of activity requirements for a $20 \mathrm{~min}$ heat in surfers using global positioning system (GPS) technology has revealed that paddling encompassed $42.6-44 \%$ of the total time with the majority of these paddling bouts $(60 \%)$ ranging from 1 to $20 \mathrm{~s}$ long $[4,5]$. This intermittent manner of surfing accentuates the importance of both the 
aerobic and anaerobic energy systems [6]. The repeated high and low intensity paddling bouts within a heat may promote a high capacity of oxygen uptake, while allowing for adequate recovery between paddling spells [3]. Meir, et al. [5] reported that during one hour of recreational surfing, the mean heart rates during arm paddling represented $80 \%$ of the laboratory peak heart rate $\left(\mathrm{HR}_{\text {peak }}\right)$ achieved by the surfers during a progressive swim bench (SWB) ergometer peak oxygen uptake $\left(\mathrm{VO}_{2 \text { peak }}\right)$ test. This suggests that a high level of aerobic fitness may be essential in surfing.

As evident through the existing body of research surrounding aerobic fitness testing in surfers, the preferred and ideal method of testing is completed with an SWB ergometer. Although dynamic leg exercise such as a cycle ergometer or treadmill are the most popular mode of exercise testing, research strongly supports the specificity of fitness testing [7]. For instance, $\mathrm{VO}_{2 \text { peak }}$ values during arm work equate to approximately $70 \%$ of the values obtained during leg exercise [8]. However, athletes with a highly trained upper-body may achieve arm-crank values approaching $90 \%$ of their cycle $\mathrm{VO}_{2 \text { peak }}$ [8].

Through a systematic review of the literature specific to aerobic fitness assessments in the surfing population, it is evident that the use of paddling ergometers to measure aerobic fitness in the elite cohort is steadily growing however remains limited in the recreational cohort $[3,6,9,10]$ when compared to mainstream sports like kayaking [11-17] (see Appendix A for search strategy).

To date, only three studies have assessed recreational surfers using SWB ergometers $[5,6,10]$. Results from these studies ranged from $31.25 \pm 6.31-54.20 \pm 10.2 \mathrm{~mL} / \mathrm{kg} / \mathrm{min}$ indicating variations in $\mathrm{VO}_{2 \text { peak }}$ across the recreational surfing cohort. Discrepancies in the results may be explained by differences in ergometers used and testing protocols employed. Previous studies measuring $\mathrm{VO}_{2 \text { peak }}$ in surfing cohorts have employed a variety of equipment like the arm crank, tether board, treadmill and bicycle, which in theory could be the result of the limited availability of SWB ergometers [18-20].

Despite the treadmill and cycle ergometer being considered the optimal form of equipment for $\mathrm{VO}_{2 \text { peak }}$ testing in both children and adults, the aforementioned differences between leg and arm work during maximal and submaximal exercise supports the use of a surfing-specific SWB ergometer $[3,7]$. However, the limited availability of SWB ergometers outside of leading sports performance institutes makes them difficult to access by recreational athletes. Given these concerns the aims of this pilot study were; to determine the $\mathrm{VO}_{2 \text { peak }}$ of recreational surfers using a new commercial SWB ergometer (Figure 1) and to propose and examine the feasibility of a regression model to predict SWB ergometer $\mathrm{VO}_{2 \text { peak }}$ values.

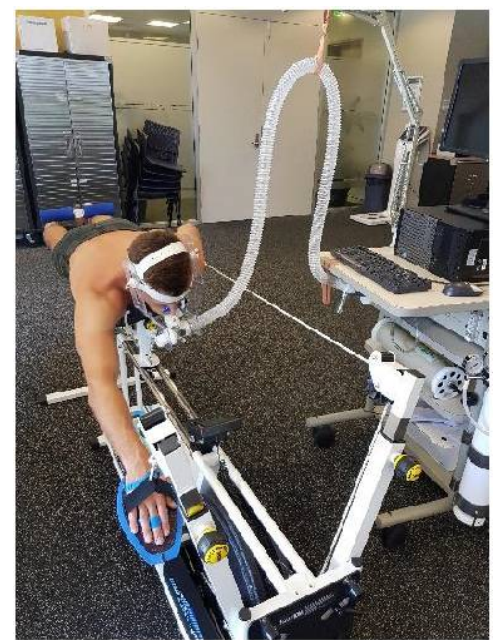

(a)

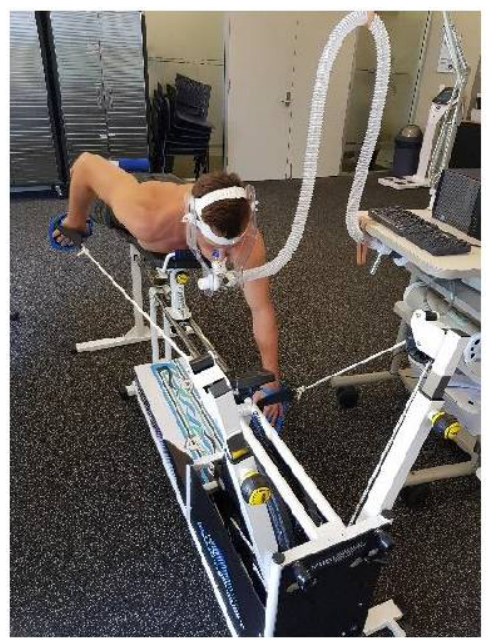

(b)

Figure 1. (a) Subject completing the catch phase of the paddling stroke on the swim bench ergometer setup in the laboratory; (b) Subject completing the pull phase of the paddling stroke on the swim bench ergometer setup in the laboratory. 


\section{Materials and Methods}

\subsection{Subjects}

This study involved nine male recreational surfers aged 18-42 years. To be classified as a recreational surfer, participants were to have a minimum of 12 months surfing experience, be currently surfing and list the sport as their main form of activity, and not be competing at higher than local club level. All testing of participants was conducted at the Bond University Institute of Health and Sport. Subjects were tested following their normal routine of sleep, nutrition and hydration levels. Ethics was granted through the Bond University Human Research Ethics Committee (RO1550) prior to commencement. Participants were informed of the associated risks and benefits of the study prior to signing an informed consent form.

\subsection{Procedures}

Testing was conducted by two experienced exercise scientists under the supervision of a senior researcher with expertise in maximal aerobic and anaerobic testing of recreational and professional surfers. Body measurements were conducted followed by the aerobic testing. All subjects underwent both a SWB ergometer and treadmill maximal oxygen consumption test. To minimize systematic bias and reduce the effects of fatigue the order of maximal oxygen consumption testing (i.e., treadmill or SWB) was randomized.

\subsection{Body Measurements}

Body measurements collected included height (EcoMed Seca, Hamburg, Germany), mass (Wedderburn, WM204, Sydney, Australia), with body mass index (BMI) subsequently calculated. Height was initially measured to the nearest $0.1 \mathrm{~cm}$ and body mass was measured to the closest $100 \mathrm{~g}$ with minimal clothing using a standard medical balance scale.

\subsection{Aerobic $\mathrm{VO}_{2 \text { peak }}$ Testing}

Prior to commencing the incremental exercise testing, subjects were provided with standardized instructions on the use of the SWB ergometer and treadmill. Subsequently subjects completed a 2-min warm-up on the apparatus they were to be tested on that day. Warm-up on the SWB ergometer included paddling at below 10 watts, while warm-up on the treadmill included running at a speed of 8 or $10 \mathrm{~km} / \mathrm{h}$ (subject preference) at an incline of 0 percent. This served to reduce the risk of injury and familiarize the subject with the equipment.

The subjects $\mathrm{VO}_{2 \text { peak }}$ was obtained using incremental exercise testing on a SWB ergometer and treadmill. Peak oxygen consumption is considered the gold-standard for quantifying aerobic fitness [21]. SWB ergometry has previously been shown to be both valid and reliable to test peak aerobic levels in both recreational and competitive surfers [6,10]. Oxygen consumption was analyzed using an automated gas analysis system (Parvo Medics, TrueOne ${ }^{\circledR}, 2400$, Sandy, UT, USA) $\left(\mathrm{O}_{2}\right.$ analyzer, $\mathrm{CO}_{2}$ analyzer, pneumotach) that was calibrated with laboratory grade standard gasses $\left(\mathrm{O}_{2}\right.$ and $\left.\mathrm{CO}_{2}\right)$ prior to each test. The SWB ergometer (KayakPro SwimFast, Miami, FL, USA) incremental test commenced at 10 watts, with increments of 10 watts every minute. This incremental test was adapted from a previously validated protocol by Furness, et al. [6]. Treadmill testing commenced at a speed of 8 or 10 $\mathrm{km} / \mathrm{h}$ based upon the subject's preference with an incline of $2 \%$ for the first minute [22]. At the start of the second minute the incline was increased to $4 \%$ then increased by $1 \%$ every subsequent minute [22]. The incremental treadmill test was developed in accordance with research by Sperlich, et al. [22] whose findings suggested that $\mathrm{VO}_{2 \text { peak }}$ scores attained by employing individually designed treadmill exercise protocols allow the athlete to pace himself or herself according to their present biological state thus achieving higher $\mathrm{VO}_{2 \text { peak }}$ values when compared with standardized protocols.

The testing termination criterion was based upon the ACSM guidelines for exercise testing and prescription [23]. Testing was terminated if age-predicted maximal heart rate was exceeded, respiratory 
exchange ratio (RER) reached greater than 1.5, oxygen consumption did not increase concurrently with power output, required power output was not maintained for greater than $10 \mathrm{~s}$, volitional exhaustion was achieved, or any symptoms of chest pain were expressed by the participant. Heart rates (HR) were monitored throughout testing via telemetry using a Polar Team Pro HR sensor (Polar H7 Bluetooth HR Sensor) connected to Polar Team Pro software, which was interfaced with the Parvo Medics system.

\subsection{Statistical Analysis}

Statistical analyses were performed using SPSS (Version 25.0; IBM Corp, Armonk, NY, USA). Normal distribution of the data was confirmed through a Shapiro-Wilks test and visual inspection of box plots, normal Q-Q plots and frequency histograms. Descriptive statistics including means $(\mathrm{M})$, standard deviations (SD) and ranges were calculated for key performance variables $\left(\mathrm{VO}_{2 \text { peak }}\right.$ $\mathrm{HR}_{\text {peak }}$, percent of age predicted $\mathrm{HR}_{\max }$ and peak aerobic power). A paired sample $t$-test was used to determine whether there was a statistical mean difference between participants $\mathrm{VO}_{2 \text { peak }}$ scores on the SWB ergometer and treadmill. A multiple regression analysis was also conducted to produce a model to predict SWB ergometer $\mathrm{VO}_{2 \text { peak }}$ scores. Prior to analyzing the data, the eight assumptions of multiple regression were considered and satisfied to ensure accuracy of the predictive model [24]. These assumptions are addressed in the results section. To assess the validity of the multiple regression model, a one-sample $t$-test was used to determine whether the mean difference between the two measures (SWB ergometer $\mathrm{VO}_{2 \text { peak }}$ values versus predicted $\mathrm{SWB}$ ergometer $\mathrm{VO}_{2 \text { peak }}$ values) were statistically different from zero. The level of agreement between the two measures was then represented through a Bland Altman plot with the associated $90 \%$ limits of agreement.

\section{Results}

\subsection{Recreational Surfer Body Measurements and Aerobic Profile}

All nine subjects successfully completed all body measurements and maximal oxygen consumption tests (treadmill and SWB) without incident. The body measurements and surfing experience of the surfers are listed in Table 1. Surfers had a mean BMI in the normal range (BMI 20.0 to $\leq 24.99 \mathrm{~kg} / \mathrm{m}^{2}$ ) and a mean surfing experience of greater than 12 years. Subjects were surfing $5.22 \mathrm{~h}$ each week. Descriptive statistics of physical attributes and experience as well as key performance variables are presented in Tables 1 and 2, respectively. Results of the paired samples $t$-tests in Table 2 demonstrate significant differences $(p<0.05)$ between the means of treadmill and SWB ergometer key performance variables.

The mean relative peak aerobic capacity for the recreational surfers on the treadmill was $66.01 \mathrm{~mL} / \mathrm{kg} / \mathrm{min}$ (range $49.0-70.1 \mathrm{~mL} / \mathrm{kg} / \mathrm{min})$, which is significantly $(p<0.001)$ greater $(+76.4 \%)$ than the SWB (M $37.41 \mathrm{~mL} / \mathrm{kg} / \mathrm{min}$, range $24.0-43.6 \mathrm{~mL} / \mathrm{kg} / \mathrm{min}$ ). Participants also had a significantly greater $(+11.8 \%, p<0.003)$ age-predicted $\mathrm{HR}_{\text {peak }}$ on the treadmill as compared to the SWB.

Table 1. Physical Attributes and Experience of Recreational Surfers $M \pm \operatorname{SD}(n=9)$.

\begin{tabular}{ll}
\hline Measure & Value \\
\hline Height $(\mathrm{cm})$ & $176.90 \pm 3.97$ \\
Mass $(\mathrm{kg})$ & $78.37 \pm 10.16$ \\
BMI $\left(\mathrm{kg} / \mathrm{m}^{2}\right)$ & $24.98 \pm 2.28$ \\
Surfing Experience (years) & $12.89 \pm 7.82$ \\
Surfing Frequency (hours per week) & $5.22 \pm 2.43$ \\
\hline
\end{tabular}


Table 2. Key Performance Variables for Recreational Surfers M ( \pm SD).

\begin{tabular}{llll}
\hline Measure & Treadmill & Swim Bench & $p$ Value \\
\hline $\mathrm{VO}_{2 \text { peak }}(\mathrm{L} / \mathrm{min})$ & $5.16( \pm 0.86)$ & $2.98( \pm 0.89)$ & $<0.001$ \\
$\mathrm{VO}_{2 \text { peak }}(\mathrm{mL} / \mathrm{kg} / \mathrm{min})$ & $66.01( \pm 8.23)$ & $37.41( \pm 8.73)$ & $<0.001$ \\
$\mathrm{VCO}_{2}$ & $5.69( \pm 1.12)$ & $3.48( \pm 0.89)$ & $<0.001$ \\
$\mathrm{VE}$ & $134.20( \pm 25.60)$ & $92.15( \pm 17.61)$ & $<0.001$ \\
$\mathrm{RQ}$ & $1.12( \pm 0.068)$ & $1.28( \pm 0.10)$ & $<0.001$ \\
$\mathrm{HR}$ & $184( \pm 10)$ & $165( \pm 14)$ & 0.004 \\
Percent of age predicted $\mathrm{HR}_{\text {peak }}(\%)$ & $98.53( \pm 4.61)$ & $88.11( \pm 7.79)$ & 0.003 \\
Peak aerobic power $(\mathrm{W})$ & $\mathrm{N} / \mathrm{A}$ & $69.91( \pm 19.33)$ & $\mathrm{N} / \mathrm{A}$ \\
\hline
\end{tabular}

\subsection{Development of a Multiple Regression Model}

Prior to generating the multiple regression model, SPSS was used to analyze the data and ensure all appropriate multiple regression assumptions were satisfied. Linearity of variables was assessed and verified by partial regression plots and a plot of studentized residuals against the predicted values. Independence of residuals was also assessed and verified by a Durbin-Watson statistic of 2.466. Homoscedasticity was verified through the visual inspection of a plot of studentized residuals versus unstandardized predicted values. There was no evidence of multicollinearity, as assessed by a tolerance value greater than 0.1 . When analyzing studentized deleted residuals values, leverage points and Cook's distances, two participants were classified as outliers and were therefore excluded from the multiple-regression analysis as recommended by Laerd Statistics [24]. When analyzing the remaining participants $(n=7)$, there was no studentized deleted residuals greater than \pm 3 standard deviations, no leverage points greater than 0.2 , and values for Cook's distance above 1 . The assumption of normality was met as previously discussed in the statistics section. The regression equation produced is presented below with a summary of the multiple regression analysis presented in Table 3. This multiple regression model predicted SWB ergometer $\mathrm{VO}_{2 \text { peak }}$ scores with an $\mathrm{R}^{2}$ value of 0.863 and an adjusted $R^{2}$ value of 0.726 .

Swim Bench $\mathrm{VO}_{2 \text { peak }}(\mathrm{mL} / \mathrm{kg} / \mathrm{min})=-33.027+(0.480 \times$ surf experience years $)+$ $(3.968 \times$ hours surfed per week $)+\left(0.699 \times\right.$ treadmill $\left.\mathrm{VO}_{2 \text { peak }}\right)$.

Table 3. Summary of Multiple Regression Analysis.

\begin{tabular}{lccc}
\hline Variable & $\mathbf{B}$ & $\mathbf{S E}_{\mathbf{B}}$ & $\boldsymbol{\beta}$ \\
\hline Intercept & -33.027 & 18.007 & \\
Surf experience (years) & 0.480 & 0.260 & 0.428 \\
Hours surfed (per week) & 3.968 & 1.616 & 0.547 \\
Treadmill VO 2 peak & 0.699 & 0.239 & 0.676 \\
$(\mathrm{~mL} / \mathrm{kg} / \mathrm{min})$ & & &
\end{tabular}

$\mathrm{B}=$ unstandardized regression coefficient; $\mathrm{SE}_{\mathrm{B}}=$ standard error of the coefficient; $\beta=$ standardized coefficient.

\subsection{Validity Assessment of SWB Ergometer Predictive Model: SWB versus Predicted SWB VO $O_{2 p e a k}$ Values}

A one-sample $t$-test determined the mean difference $(0.0084 \mathrm{~mL} / \mathrm{kg} / \mathrm{min})$ between the two measures (SWB ergometer $\mathrm{VO}_{2 \text { peak }}$ values versus predicted $\mathrm{SWB}$ ergometer $\mathrm{VO}_{2 \text { peak }}$ values) was not statistically $(p>0.05)$ different from zero. Bland-Altman plots with the associated $90 \%$ limits of agreement (5.83 and -5.79) are presented in Figure 2. 


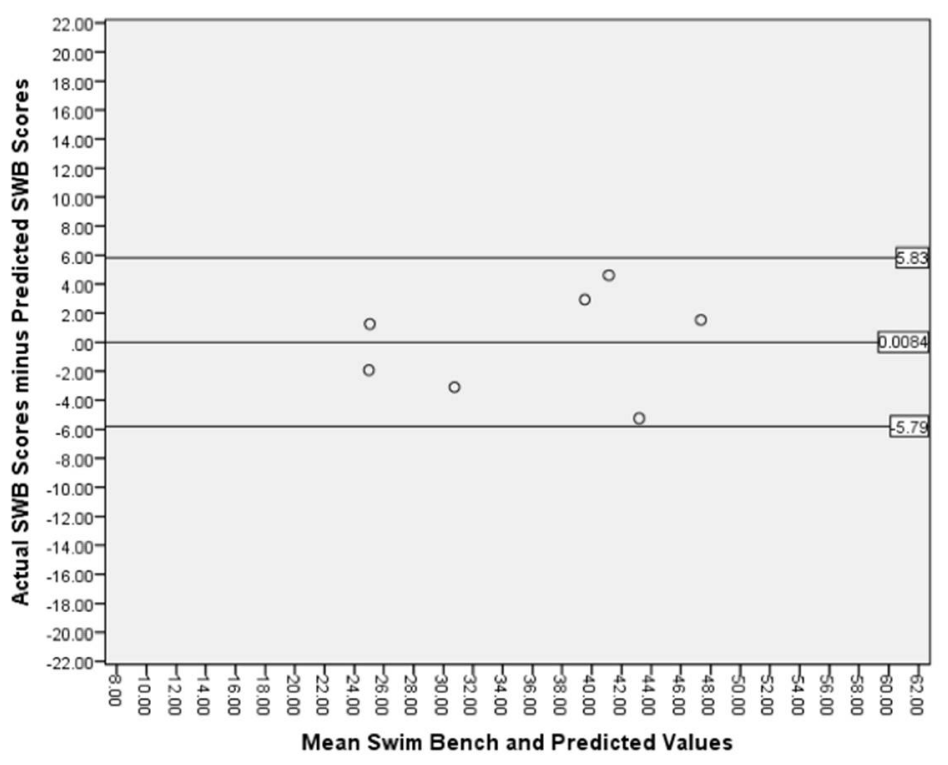

Figure 2. Bland Altman Plot displaying mean difference between the two measures and 90\% CI.

\section{Discussion}

The purpose of this study was to determine the $\mathrm{VO}_{2 \text { peak }}$ of recreational surfers using a new commercial SWB ergometer and to propose a regression model to predict SWB ergometer $\mathrm{VO}_{2 \text { peak }}$. Both aims have been satisfied and are discussed in detail below.

\subsection{Body Measurements}

This study has provided a profile of the recreational surfing cohort, which included body measurements of height, mass and key performance variables $\left(\mathrm{HR}_{\text {peak }}, \mathrm{VO}_{2 \text { peak }}\right.$, aerobic power output). Participants in this study had a mean age of $32.78( \pm 6.91)$ years versus $18( \pm 2.0)$ and $21.2( \pm 2.7)$ by Loveless and Minahan [10] and Meir, et al. [5] respectively. With respect to height of the male recreational surfing population, the mean height in the present study was $176.90( \pm 3.97) \mathrm{cm}$, which was slightly less than previous studies. Loveless and Minahan [10] investigated eight recreational surfers and reported a mean height of $179( \pm 11.0) \mathrm{cm}$. This is similar to the mean height of $180.13( \pm 7.54) \mathrm{cm}$ reported by Furness, et al. [6] in 47 recreational surfers.

Mean body mass presented with similarities and discrepancies to previous research. In the present study, male recreational surfers had a mean body mass of $78.37( \pm 10.16) \mathrm{kg}$ which was similar to the mean body mass of $77.42( \pm 10.69) \mathrm{kg}$ reported by Furness, et al. [6] (77.42 \pm 10.69$)$. Previous research by Meir, et al. [5] and Loveless and Minahan [10] found mean mass to be slightly less with the authors reporting values of $68.9( \pm 5.67) \mathrm{kg}$ and $66.8( \pm 13.00) \mathrm{kg}$ respectively. The discrepancy in mean body mass reported in the present study and those by Meir, et al. [5] and Loveless and Minahan [10] could potentially be attributed to body composition changes that occur with aging such as increased fat mass [25].

\subsection{Aerobic Profiles}

Surfing is an activity characterized by intermittent exercise bouts of varying intensities and durations that requires exceptional aerobic fitness [3]. The use of time-motion analysis to monitor the activity requirements of a 20-min heat in surfers using GPS technology has revealed that paddling bouts account for $42.6-44 \%$ of the heat emphasizing the importance of the aerobic energy system in surfers $[4,5]$.

The findings from this study established that recreational surfers had mean $\mathrm{VO}_{2 \text { peak }}( \pm \mathrm{SD})$ values of $66.01( \pm 8.23)$ and $37.41( \pm 8.73) \mathrm{mL} / \mathrm{kg} / \mathrm{min}$ on the treadmill and SWB respectively. When these 
values were compared to those of recreational runners and swimmers it was found that recreational surfers were capable of achieving larger $\mathrm{VO}_{2 \text { peak }}$ values on the treadmill. Research by Gillen, et al. [26] assessed 11 recreational runners with a mean age of 34.1 years and found them to have a mean $\mathrm{VO}_{2 \text { peak }}$ of $58.4 \pm 7.8 \mathrm{~mL} / \mathrm{kg} / \mathrm{min}$ which is less than the mean value of this study's recreational surfing cohort. Kimura, et al. [27] found recreational swimmers to have a mean $\mathrm{VO}_{2 \text { peak }}$ of $58.3 \pm 4.2 \mathrm{~mL} / \mathrm{kg} / \mathrm{min}$ on a treadmill, which was also less than the values achieved by the recreational surfing cohort in this study. It is theorized that surfing's intermittent nature of high and low intensity bouts influence adaptions in physiological variables that impact aerobic endurance performance such as maximal oxygen uptake $\left(\mathrm{VO}_{2 \text { peak }}\right)$, lactate threshold, and work economy $[28,29]$. It has been demonstrated by previous research that high-aerobic intensity training otherwise known as high intensity interval training (HIIT) results in significantly increased absolute $\mathrm{VO}_{2 \text { peak }}$ values when compared to alternative training methods such as long slow distance running and lactate running [29,30].

The SWB ergometer findings have similarities [10] and discrepancies [5,6] with previous research conducted on recreational surfing cohorts. The SWB ergometer $\mathrm{VO}_{2 \text { peak }}$ values from this study are similar to those reported by Loveless and Minahan [10] $(37.8 \pm 4.5 \mathrm{~mL} / \mathrm{kg} / \mathrm{min})$. These findings differ to those reported by Meir, et al. [5] $(54.20 \pm 10.2 \mathrm{~mL} / \mathrm{kg} / \mathrm{min})$ and greater than those reported by Furness, et al. [6] ( $37.41 \pm 8.73 \mathrm{~mL} / \mathrm{kg} / \mathrm{min})$. The authors of this study hypothesize that the differences in $\mathrm{VO}_{2 \text { peak }}$ could be attributed to the type of SWB ergometer used. The SwimFast ergometer used in this study differs from the Vasa and Repco ergometers used in previous studies as it allows for torsional roll $\left(\approx 30^{\circ}\right)$, which enables the recruitment of additional muscle groups in the thoracic region. This recruitment of additional muscle groups could lead to an increase in oxygen consumption resulting in higher $\mathrm{VO}_{2 \text { peak }}$ values. The effects of the torsional roll on $\mathrm{VO}_{2 \text { peak }}$ are further evident when examining the mean age of the cohorts used in the present study and previous research. The mean age for the present study was $32.78( \pm 6.91)$ yrs compared to younger subjects of $18.0 \mathrm{yrs}( \pm 2.0)$ and $26.50 \mathrm{yrs}$ ( \pm 5.28$)$ by Loveless and Minahan [10] and Furness, et al. [6], respectively. Aging is typically associated with a progressive decline in the capacity for physical activity due to the reduction in maximal rate of oxygen utilization [31]. However, as evident by the findings of this study, the increased age of our cohort did not result in reduced $\mathrm{VO}_{2 \text { peak }}$ values when compared to the studies by Loveless and Furness. This further supports the theory that the torsional roll of the SwimFast ergometer allows for the use of larger muscle groups and consequently resulting in a greater need for oxygen.

To date, limited research has compared $\mathrm{VO}_{2 \text { peak }}$ values obtained from $\mathrm{SWB}$ ergometer to those obtained from a treadmill [18]. When comparing $\mathrm{VO}_{2 \text { peak }}$ from tethered board paddling, prone hand cranking and treadmill running, Lowdon, et al. [18] found that the peak oxygen consumption produced by running on a treadmill were the greatest. Previous research has demonstrated that aerobic capacity during arm work was considerably lower than utilizing the legs [8,32,33]. Stenberg, et al. [33] compared arm work to leg work and found that $\mathrm{VO}_{2 \text { peak }}$ during arm ergometry was only $66 \%$ of that achieved by sitting leg cycle ergometry. This relates to the principle of specificity which states that exercise of a specific type, intensity and duration that utilizes a specific muscle group will elicit distinct physiological adaptions which in turn highlights the importance of sport-specific testing [18]. When using lower limb dominant equipment such as a treadmill or a cycle ergometer, the use of the larger leg muscles will demand greater oxygen consumption than the smaller upper limb muscles therefore resulting in a greater $\mathrm{VO}_{2 \text { peak }}[3,8,18,32]$. From this previous research, it can be deduced that even within the same person peak oxygen uptake is specific to a given type of activity. Therefore, to obtain relevant values, emphasis should be placed on the testing methods and its specificity to the activity of the participant. Consequently, when assessing the $\mathrm{VO}_{2 \text { peak }}$ of surfers the SWB should be the standard and preferred testing method.

Peak aerobic power outputs in this study were found to be less than previous studies. The mean peak aerobic power output $(\mathrm{W})$ in this study was $69.91( \pm 19.33) \mathrm{W}$ which was less than the 101.26 ( \pm 18.49$) \mathrm{W}$ and $199( \pm 24) \mathrm{W}$ reported by Furness, et al. [6] and Loveless and Minahan [10], respectively. The authors hypothesize the discrepancies in peak aerobic power outputs may be 
attributed to the differences in computation algorithms used by the SwimFast and Vasa ergometers however this was outside the scope of this study.

Taking into account the demanding activity of arm paddling in surfing, Meir, et al. [5] reported that during one hour of recreational surfing, the mean HRs during arm paddling represented $80 \%$ of the laboratory $\mathrm{HR}_{\text {peak }}$ attained by the surfers during a progressive $\mathrm{SWB}$ ergometer $\mathrm{VO}_{2 \text { peak }}$ test.

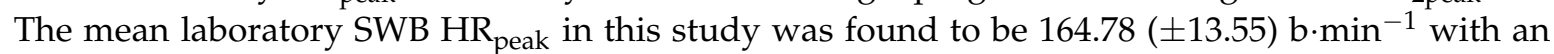
associated mean percent of age-predicted $\mathrm{HR}_{\text {peak }}$ of $88.11 \%$ ( \pm 7.79 ) (Table 2). Meir, et al. [5] reported a mean $\mathrm{HR}_{\text {peak }}$ of $180( \pm 6) \mathrm{b} \cdot \mathrm{min}^{-1}$ and mean percent of age-predicted $\mathrm{HR}_{\text {peak }}$ of approximately 90.5\%. The results by Meir, et al. [5] compared favorably to Loveless and Minahan [10] who reported a mean $\mathrm{HR}_{\text {peak }}$ of $194( \pm 5) \mathrm{b} \cdot \mathrm{min}^{-1}$ and mean percent of age-predicted $\mathrm{HR}_{\text {peak }}$ of approximately $96 \%$. Furness, et al. [6] reported a mean peak HR of $175.58( \pm 10.51) \mathrm{b} \cdot \mathrm{min}^{-1}$ with an approximate percent of age-predicted $\mathrm{HR}_{\text {peak }}$ of $90.74 \%$. The similarities in values of mean percent of age-predicted $\mathrm{HR}_{\text {peak }}$ between the present study and previous research points to similarities between the protocols used in terms of eliciting similar cardiovascular demands on the participants.

\subsection{Multiple Regression Model}

The final aim of this study was to propose a regression model to predict SWB ergometer $\mathrm{VO}_{2 \text { peak }}$ values. This regression model would serve to provide recreational surfers with no access to an SWB ergometer the opportunity to predict their surfing specific $\mathrm{VO}_{2 \text { peak }}$.

After satisfying the eight assumptions of a multiple regression analysis, it was determined that the three independent variables (predictors) best included in the model were: (1) treadmill $\mathrm{VO}_{2 \text { peak }}$ ( $\mathrm{mL} / \mathrm{kg} / \mathrm{min})$; (2) surf experience (years); and (3) hours surfed (per week). The models $\mathrm{R}^{2}$ value of 0.863 infers that these three independent variables can explain $86.3 \%$ of the variability in the dependent variable (SWB ergometer $\mathrm{VO}_{2 \text { peak }}$ ). The model's adjusted $\mathrm{R}^{2}$ value of 0.726 corrects for positive bias to provide a value that would be expected in the population. Therefore, when generalized to a larger population, the independent variables in this model explain $72.6 \%$ of the variability in the dependent variable. Given the development of this equation, further research should aim to conduct profiling on a larger recreational cohort to further assess its validity.

\section{Study Limitations and Strengths}

This pilot study is the first to explore the novel idea of producing a regression model to predict surf specific $\mathrm{SWB} \mathrm{VO}_{2 \text { peak }}$ values in a recreational surfing cohort. This study acts as a requisite initial step in exploring the use of such a regression model in a larger recreational surfing cohort and paves the path for further study into producing a similar model for a larger competitive surfing cohort. In addition to being the first pilot study to produce a regression model, this study is one of few to profile recreational surfers on both a SWB ergometer and a treadmill.

The authors acknowledge the regression model produced is not without limitations and further study is warranted in the area. It is important to note that the model is only relevant to the current sample and therefore caution should be exercised when using this model in age groups and surfing populations outside of the current study. Further research on a significantly larger sample size is necessary to validate the regression model to allow generalization and application outside of this study.

\section{Conclusions}

The present study is the first to use the SwimFast ergometer in $\mathrm{VO}_{2 \text { peak }}$ testing in recreational surfers and compare its values to those from the gold-standard treadmill within the same sample. The authors propose that the results will help in bolstering the current knowledge of available tools for physiological assessments in the surfing population. Furthermore, the present study was the first to produce a regression model for predicting SWB ergometer $\mathrm{VO}_{2 \text { peak }}$. The regression model which uses surfing experience (years), hours surfed (per week) and treadmill $\mathrm{VO}_{2 \text { peak }}$ values $(\mathrm{mL} / \mathrm{kg} / \mathrm{min}$ ) could allow surfers with no access to an SWB ergometer to predict their surfing specific $\mathrm{VO}_{2 \text { peak }}$. This could 
allow the surfer to compare their peak aerobic fitness with other recreational and elite surfers allowing them to identify strengths or deficiencies in their aerobic power and potential goals for improvement.

Author Contributions: J.F. and W.H. conceived and designed the experiments; H.K. and E.S. performed the experiments; H.K. and J.F. analyzed the data; H.K. wrote the paper; J.F., M.C., B.S. and W.H. reviewed and provided feedback for approval of the final manuscript draft.

Funding: This research received no external funding.

Acknowledgments: The researchers would like to acknowledge the generosity of KayakPro for the SWB ergometer utilized to conduct all SWB $\mathrm{VO}_{2 \text { peak }}$ testing throughout this study.

Conflicts of Interest: The authors declare no conflicts of interest.

\section{Appendix}

Literature review and appraisal were conducted using the search terms "Surf*", "Cardiopulmonary Fitness", " $\mathrm{VO}_{2 \max }$ ", "Peak $\mathrm{VO}_{2}$ ", "Ergometer" and "Treadmill". These search terms were combined through the use of Boolean operators "AND" and "OR" to limit and refine the search. This search provided an awareness of the existing literature on the topic and was used to inform the formal search criteria.

A two-tiered search strategy was then implemented. First, a comprehensive search of online databases including PubMed, CINHAL, SPORTSDiscus, and EBSCO was completed. An identical search string was used in each database, and no limits were applied. Search results were compiled in Endnote, and duplicates removed. Second, reference lists of key articles and reviews identified by the search were pearled in order to identify additional relevant papers. All articles were screened for inclusion using the following defined criteria: (1) published in English; (2) involved human participants; (3) participants mean age $>18$ years; (4) participants $>12$ months experience in surfing; (5) the article specifically investigated $\mathrm{VO}_{2 \text { peak }}$ using an ergometer or treadmill (6) oxygen consumption was analyzed using a gas analysis system meeting the Australian Institute of Sport accreditation standards for precision and accuracy; (7) the article contained original data. For this study, $\mathrm{VO}_{2 \text { peak }}$ was defined as the highest rate at which oxygen could be taken up and consumed by the body during intense exercise. Ergometer was defined as an apparatus that measures work and energy expenditure during a period of physical exercise.

The methodological quality of the articles selected for inclusion was assessed using the Joanna Briggs Institute (JBI) Appraisal Checklist for Cohort Studies. The JBI Appraisal Checklist employs an 11-question checklist to determine the extent to which the study has addressed the possibility of bias in its design, conduct and analysis [34]. The checklist is comprised of closed answer questions where a "yes" is awarded 1 point and a "no" or "unclear" is awarded 0 points. A modified version of this tool was used where questions 2, 7, 8, 9 and 10 were removed, as they were designed to evaluate aspects of methodological quality that did not align with the design of the included articles. All studies were independently rated by the authors with the level of agreement measured using a Cohen's Kappa analysis of all raw scores (6 scores per paper). For final scores, any disagreements in points awarded were settled by consensus. Due to the modifications made to the JBI checklist, all scores were first converted to percentages to enable grading as proposed by Lyons, et al. [35]. On this basis, the grading criteria applied when rating the methodological quality of the included studies were as follows: JBI total score $<61 \%$, 'poor' methodological quality; $>61 \%$, 'good' methodological quality. Following the critical appraisal of the included studies, key data were extracted and tabulated from all included studies.

Search String for PubMed, CINHAL, SPORTSDiscus, and EBSCO: (((cardiopulmonary response OR cardiovascular response OR oxygen consumption OR physiological impact OR physiological capacity OR physiological response OR physiological effects OR physiological profile OR maximal aerobic power OR aerobic OR performance OR physiological Parameter* OR peak power output)) AND (treadmill OR maximal exercise testing OR graded exercise testing OR high intensity testing OR 
$\mathrm{VO}_{2 \text { max }}$ test OR $\mathrm{VO}_{2 \max }$ OR $\mathrm{VO}_{2 \text { peak }}$ test OR $\mathrm{VO}_{2 \text { peak }}$ OR swim bench OR ergometer OR ergometers OR ergometric)) AND (Surf*).

\section{References}

1. Meir, R.A.; Shi, Z.; Wendy, L.G.; Rosanne, A.C. An investigation of surf injury prevalence in Australian surfers: A self-reported retrospective analysis. N. Z. J. Sports Med. 2012, 39, 52-58.

2. Moran, K.; Webber, J. Surf, sand, scrapes and stings: First aid incidents involving children at New Zealand beaches, 2007-2012: Childhood beach injuries. J. Paediatr. Child Health 2014, 50, 221-225. [CrossRef] [PubMed]

3. Mendez-Villanueva, A.; Bishop, D. Physiological aspects of surfboard riding performance. Sports Med. 2005, 35, 55-70. [CrossRef] [PubMed]

4. Secomb, J.L.; Sheppard, J.M.; Dascombe, B.J. Time-motion analysis of a 2-hour surfing training session. Int. J. Sports Physiol. Perform. 2015, 10, 17-22. [CrossRef] [PubMed]

5. Meir, R.; Lowdon, B.; Davie, A. Heart rates and estimated energy expenditure during recreational surfing. J. Sci. Med. Sport 1991, 23, 70-74.

6. Furness, J.; Hing, W.; Sheppard, J.M.; Newcomer, S.; Schram, B.; Climstein, M. The Physiological Profile of Male Competitive And Recreational Surfers. J. Strength Cond. Res. 2016, 32, 372-378. [CrossRef] [PubMed]

7. Loftin, M.; Sothern, M.; Warren, B.; Udall, J. Comparison of $\left.\mathrm{VO}_{2}\right)$ Peak during Treadmill and Cycle Ergometry in Severely Overweight Youth. J. Sports Sci. Med. 2004, 3, 554-560. [PubMed]

8. Sawka, M.N. Physiology of upper body exercise. Exerc. Sport Sci. Rev. 1986, 14, 174-211. [CrossRef]

9. Farley, O.; Harris, N.K.; Kilding, A.E. Anaerobic and aerobic fitness profiling of competitive surfers. J. Strength Cond. Res. 2012, 26, 2243-2248. [CrossRef] [PubMed]

10. Loveless, D.J.; Minahan, C. Peak aerobic power and paddling efficiency in recreational and competitive junior male surfers. Eur. J. Sport Sci. 2010, 10, 407-415. [CrossRef]

11. Van Someren, K.A.; Phillips, G.R.; Palmer, G.S. Comparison of physiological responses to open water kayaking and kayak ergometry. Int. J. Sports Med. 2000, 21, 200-204. [CrossRef] [PubMed]

12. Jones, M.J.; Peeling, P. A Comparison of Laboratory-Based Kayak Testing Protocols. Int. J. Sports Physiol. Perform. 2014, 9, 346-351. [CrossRef] [PubMed]

13. Forbes, S.C.; Chilibeck, P.D. Comparison of a kayaking ergometer protocol with an arm crank protocol for evaluating peak oxygen consumption. J. Strength Cond. Res. 2007, 21, 1282-1285. [PubMed]

14. Fry, R.W.; Morton, A.R. Physiological and kinanthropometric attributes of elite flatwater kayakists. Med. Sci. Sports Exerc. 1991, 23, 1297-1301. [CrossRef] [PubMed]

15. Gomes, B.B.; Mourão, L.; Massart, A.; Figueiredo, P.; Vilas-Boas, J.P.; Santos, A.M.C.; Fernandes, R.J. Gross Efficiency and Energy Expenditure in Kayak Ergometer Exercise. Int. J. Sports Med. 2012, 33, 654-660. [CrossRef] [PubMed]

16. Billat, V.; Faina, M.; Sardella, F.; Marini, C.; Fanton, F.; Lupo, S.; Faccini, P.; de Angelis, M.; Koralsztein, J.P.; Dalmonte, A. A comparison of time to exhaustion at $\mathrm{VO}_{2}$ max in elite cyclists, kayak paddlers, swimmers and runners. Ergonomics 1996, 39, 267-277. [CrossRef] [PubMed]

17. Billat, L.V.; Koralsztein, J.P. Significance of the velocity at $\mathrm{VO}_{2 \max }$ and time to exhaustion at this velocity. Sports Med. 1996, 22, 90-108. [CrossRef] [PubMed]

18. Lowdon, B.J.; Bedi, J.F.; Horvath, S.M. Specificity of aerobic fitness testing of surfers. Aust. J. Sci. Med. Sport 1989, 21, 7-10.

19. Lowdon, B.; Pateman, A. Physiological Parameters of International Surfers. Aust. J. Sci. Med. Sport 1980, 12, 30-33.

20. Tesch, P.A. Physiological characteristics of elite kayak paddlers. Can. J. Appl. Sport Sci. 1983, 8, 87-91. [PubMed]

21. Seiler, S. A brief history of endurance testing in athletes. Sportscience 2011, 15, 40-86.

22. Sperlich, P.F.; Holmberg, H.-C.; Reed, J.L.; Zinner, C.; Mester, J.; Sperlich, B. Individual versus Standardized Running Protocols in the Determination of $\mathrm{VO}_{2}$ max $)$. J. Sport Sci. Med. 2015, 14, 386-393.

23. Baechle, T.R.; Earle, R.W. Essentials of Strength Training and Conditioning; Human Kinetics: Champaigne, IL, USA, 2008.

24. Statistics, L. Paired-Samples T-Test Using SPSS Statistics. Available online: https://statistics.laerd.com/ (accessed on 7 February 2018). 
25. St-Onge, M.P.; Gallagher, D. Body composition changes with aging: The cause or the result of alterations in metabolic rate and macronutrient oxidation? Nutrition 2010, 26, 152-155. [CrossRef] [PubMed]

26. Gillen, Z.M.; Wyatt, F.B.; Winchester, J.B.; Smith, D.A.; Ghetia, V. The Relationship between Aerobic and Anaerobic Performance in Recreational Runners. Int. J. Exer. Sci. 2016, 9, 625-634.

27. Kimura, Y.; Yeater, R.A.; Martin, R.B. Simulated swimming: A useful tool for evaluation the $\mathrm{VO}_{2 \max }$ of swimmers in the laboratory. Br. J. Sports Med. 1990, 24, 201-206. [CrossRef] [PubMed]

28. Pate, R.R.; Kriska, A. Physiological basis of the sex difference in cardiorespiratory endurance. Sports Med. 1984, 1, 87-98. [CrossRef] [PubMed]

29. Helgerud, J.; Hoydal, K.; Wang, E.; Karlsen, T.; Berg, P.; Bjerkaas, M.; Simonsen, T.; Helgesen, C.; Hjorth, N.; Bach, R.; Hoff, J. Aerobic high-intensity intervals improve $\mathrm{VO}_{2 \max }$ more than moderate training. Med. Sci. Sports Exerc. 2007, 39, 665-671. [CrossRef] [PubMed]

30. Upadhyay, V.; Chowdhery, A.; Bhattacharyya, M. Effect of high intensity interval training and slow, continuous training on $\mathrm{VO}_{2 \max }$ of school going non-athlete males: A comparative study. Br. J. Sports Med. 2010, 44, i19. [CrossRef]

31. Betik, A.C.; Hepple, R.T. Determinants of $\mathrm{VO}_{2}$ max decline with aging: An integrated perspective. Appl. Physiol. Nutr. Metab. 2008, 33, 130-140. [CrossRef] [PubMed]

32. Asmussen, E.; Hemmingsen, I. Determination of maximum working capacity at different ages in work with the legs or with the arms. Scand. J. Clin. Lab. Investig. 1958, 10, 67-71. [CrossRef] [PubMed]

33. Stenberg, J.; Astrand, P.O.; Ekblom, B.; Royce, J.; Saltin, B. Hemodynamic response to work with different muscle groups, sitting and supine. J. Appl. Physiol. 1967, 22, 61-70. [CrossRef] [PubMed]

34. Institute, J.B. The Joanna Briggs Institute Critical Appraisal Tools for Use in JBI Systematic Reviews Checklist for Cohort Studies Institute; The Joanna Briggs Institure: Adelaide, Australia, 2017.

35. Lyons, K.; Radburn, C.; Orr, R.; Pope, R. A Profile of Injuries Sustained by Law Enforcement Officers: A Critical Review. Int. J. Environ. Res. Public Health 2017, 14, 142. [CrossRef] [PubMed] 